\title{
Surface-magnetoplasmon nonreciprocity effects in noble-metal/ferromagnetic heterostructures
}

\author{
J. B. González-Díaz, ${ }^{1}$ A. García-Martín, ${ }^{1}$ G. Armelles, ${ }^{1}$ J. M. García-Martín, ${ }^{1}$ C. Clavero, ${ }^{1}$ A. Cebollada, ${ }^{1,2}$ R. A. Lukaszew, ${ }^{3}$ \\ J. R. Skuza, ${ }^{3}$ D. P. Kumah, ${ }^{2}$ and R. Clarke ${ }^{2}$ \\ ${ }^{1}$ Instituto de Microelectrónica de Madrid, Consejo Superior de Investigaciones Científicas, Isaac Newton 8, Tres Cantos, \\ E-28760 Madrid, Spain \\ ${ }^{2}$ FOCUS Center, University of Michigan, Ann Arbor, Michigan 48109, USA \\ ${ }^{3}$ Physics and Astronomy Department, University of Toledo, Mstop 111, 2801 W. Bancroft, Toledo, Ohio 43606, USA
}

(Received 6 August 2007; published 5 October 2007)

\begin{abstract}
We demonstrate magnetic field control of surface plasmon excitations in noble-metal/ferromagnetic/noble metal trilayers, analogous to the effects previously observed in semiconductor structures. We show that the coupling of an external magnetic field to the surface plasmon-polariton wave vector is greatly enhanced in the metallic structure due to the ferromagnetic nature of one of its constituents. The observed coupling could be used to modulate the surface plasmon response in ultrasensitive spectroscopic applications.
\end{abstract}

DOI: 10.1103/PhysRevB.76.153402

PACS number(s): 78.20.Ls, 73.20.Mf, 78.66.Bz, 42.25.Bs

Surface plasmon-polariton (SPP) modes are electromagnetic excitations localized at the interface between two media, one with positive and the other with negative dielectric constant. These modes may appear at the interface between a degenerate semiconductor and a dielectric or between a metal and a dielectric. In the former case, due to the low value of the plasma frequency of the semiconductor, the frequencies of the SPPs are restricted to the far infrared range, whereas in the second case the SPP modes can have frequencies varying from the far infrared to the visible range. The propagation characteristics of the SPPs and their EM field distribution depend strongly on the optical properties and interface morphology of the system. This dependence has been exploited in different optical contexts such as light guiding at the subwavelength scale, ${ }^{1-3}$ optical switching, ${ }^{4}$ biochemical sensing, ${ }^{5}$ or nanometer resolved far-field optical microscopy. ${ }^{6}$

To date SPPs are commonly considered as passive, i.e., insensitive to the magnetic field and just depending on the optical and geometrical properties of the system. In this work we demonstrate the control of SPP excitations in metallic trilayer structures by means of an external magnetic field. We show that the coupling of the magnetic field to the wave vector of the plasmon is greatly enhanced by the ferromagnetic nature of the trilayer structure. This effect was first studied theoretically in semiconductor-based SPPs $^{7-9}$ and in metals. ${ }^{10}$ The effect of the magnetic field on the properties of the SPP modes depends on the relative orientation of the applied magnetic field with respect to the wave vector of the SPP. In particular, we will show that when the magnetic field is applied perpendicular to the direction of propagation of the SPP and parallel to the interface, it modifies the dispersion relation of the SPP mode in such a way that the dispersion relation depends on the $k$ direction [i.e., $w(k) \neq w(-k)$ ]. Experimentally this magnetic field induced nonreciprocity has been observed on semiconductor-based SPPs, ${ }^{11}$ but not yet in metallic systems. This is due to the high magnetic field needed to observe magnetic field induced effects on metallic based SPPs.

One way to reduce the required external magnetic field is to incorporate ferromagnetic metals. Due to the magneto- optical (MO) activity that many ferromagnetic materials exhibit at low magnetic fields, surface magnetoplasmon effects could be more easily observable in ferromagnetic metals. However, due to the high values of their absorption losses, the SPP features are significantly broadened, making this approach difficult, if not impractical. A solution to this problem is suggested by multilayer configurations which combine both noble-metal and ferromagnetic layers. ${ }^{12,13}$ In these systems, which consist of sandwiches of $\mathrm{Au} / \mathrm{Co} / \mathrm{Au}$ layers, a strong enhancement of the MO properties has been observed and attributed to SPP excitation. The ferromagnetic layer induces MO activity which is not observable in the noblemetal layer alone. These hybrid systems are strong candidates to observe magnetic field induced nonreciprocal effects in metallic-based SPP structures.

We will use these hybrid systems to show that nonreciprocal surface magnetoplasmon effects can also be observed in metallic-based SPPs. This proves that the external magnetic field not only acts on the magnetic counterpart of the structure, but also on the plasmon excitation itself. To do so we have analyzed the transverse Kerr effect in $\mathrm{Au} / \mathrm{Co} / \mathrm{Au}$ layered structures as a function of Co thickness and SPP excitation conditions. We show that when the SPP is excited in these structures the external magnetic field induces a modification of the coupling of the incident light with the SPP, leading to a transverse Kerr effect. We show that this modification of the light-SPP coupling reflects a nonreciprocity effect in the SPP propagation, which is induced by the magnetization of the ferromagnetic layer and can be tuned by modifying the thickness of the ferromagnetic layer. The nonreciprocity in the SPP propagation may have important consequences in the design of new plasmonic devices based on nanostructured metallic films, as the magnetization could control the propagation properties and coupling mechanism of the SPP modes.

A series of $6 \mathrm{~nm} \mathrm{Au} / \mathrm{Co} / 16 \mathrm{~nm} \mathrm{Au/glass} \mathrm{structures} \mathrm{were}$ grown by molecular beam epitaxy. The Co thickness was varied between 0 and $9 \mathrm{~nm}$. Complete details of the deposition conditions, and optimization of the structure and morphology of the samples can be found elsewhere. ${ }^{14}$ To excite the SPP in these structures we have used a Kretschmann configuration, ${ }^{15,16}$ where the sample is optically interfaced to 


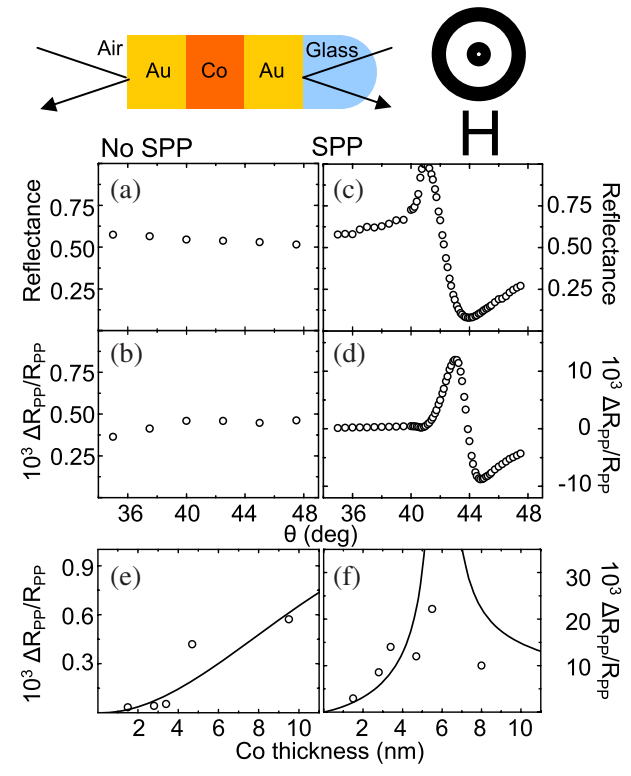

FIG. 1. (Color online) Top: schematic view of the sample configuration. If the light is incident from the left, no SPP excitation occurs; if light is incident from the right the prism allows SPP excitation (Kretschman configuration). [(a)-(d)] Experimental behavior of the reflectivity $R_{p p}$ and transverse Kerr signal $\Delta R_{p p} / R_{p p}$ as a function of the incidence angle for the $4.7 \mathrm{~nm}$ Co trilayer sample. No surface plasmon modes are excited in the left hand panels, whereas the right hand panels show the corresponding data with SPP excitation; (e) and (f) correspond to experimental (dots) and theoretical values (solid line, see text) of the transverse Kerr signal intensity as a function of the Co layer thickness, without (e) and with (f) SPP excitation.

a half-cylinder glass prism by a matching index fluid, placing the glass substrate in contact with the prism and leaving the Au capping layer in contact with air. The prism is mounted in a goniometer permitting variation of the light incidence angle from a fixed TM polarized HeNe laser $(632 \mathrm{~nm})$. In this way we can excite the SPP of the $\mathrm{Au} / \mathrm{Co} / \mathrm{Au} /$ glass structure with a maximum of the EM field at the Au/air interface.

Placing the sample at the center of a pair of Helmholtz coils, an external magnetic field is applied in the plane of the sample and perpendicular to the plane of incidence. This is the geometry appropriate to the transverse magneto-optic Kerr effect (TMOKE). The reflected light is detected by a $\mathrm{Si}$ photodiode which has a $p$-oriented polarizer in front of it. To obtain comparative results without any SPP excitation the TMOKE signal was also measured with the same experimental geometry but without the glass prism. Due to thickness dependence of the out-of-plane vs in-plane anisotropy of the $\mathrm{Au} / \mathrm{Co}$ system, the magnetic field needed to saturate the sample in the plane depends on the Co thickness. For the samples with Co thickness less than $2.5 \mathrm{~nm}$ we needed a magnetic field of $0.5 \mathrm{~T}$, whereas for higher Co thickness magnetic saturation was achieved at a magnetic field of $0.005 \mathrm{~T}$.

In Fig. 1 we show the angular dependence of the reflectivity in the demagnetized state $\left(R_{p p}\right)$ and the normalized magnetic field induced variation of the reflectivity, $\left[\Delta R_{p p} / R_{p p}\right.$, where $\Delta R_{p p}\left(m_{y}\right)=R_{p p}(+1)-R_{p p}(-1), m_{y}$ the $y$ component of the normalized magnetization $\left(m_{y}=M_{y} / M_{s}\right)$ and $M_{s}$ is the magnetization of the Co layer at saturation] without [Figs. 1(a) and 1(b)] and with [Figs. 1(c) and 1(d)] SPP excitation for a specific Co thickness of $4.7 \mathrm{~nm}$. The variation of both $R_{p p}$ and $\Delta R_{p p} / R_{p p}$ as a function of the incident angle $\theta$ shows a smooth featureless evolution in the absence of SPP excitation, in contrast with the behavior when the SPP is excited. The latter situation reveals significant structure in both $R_{p p}$ and $\Delta R_{p p} / R_{p p}$ for incident angles in the range of $40^{\circ}-50^{\circ}$.

Let us first focus on the angular dependence of $\Delta R_{p p} / R_{p p}$. If the SPP is not excited, $\Delta R_{p p} / R_{p p}$ is due to the modification of the EM field induced by the nondiagonal component of the dielectric tensor of Co (standard TMOKE), which gives rise to a smooth angular dependence of $\Delta R_{p p} / R_{p p}$. However, when the SPP is excited, the angular dependence of $\Delta R_{p p} / R_{p p}$ is quite different. In particular, there is a strong increase in the values of $\Delta R_{p p} / R_{p p}$, for instance, at $\sim 43 \mathrm{deg}$, $\Delta R_{p p} / R_{p p}$ is of the order of $0.5 \times 10^{-3}$ [Fig. 1(b)] without SPP excitation but reaches $1 \times 10^{-2}$ when SPP is excited [Fig. 1(d)]. Also, the shape of the curve is similar to the derivative of the reflectivity curve with respect to the incident angle. This suggests that, in addition to the modification of the EM field induced by the nondiagonal component of the dielectric tensor of $\mathrm{Co}$, other mechanisms contribute to the magnetic field induced variation of the reflectivity when the SPP is excited. In fact, a magnetic field applied in the plane of the structure and perpendicular to the SPP propagation direction modifies the SPP wave vector, and this modification indeed plays an important a role in the magnetic field induced variation of the reflectivity, as will be shown.

A simple closed-form expression of the magnetic field dependence of the SPP wave vector for the $\mathrm{Au} / \mathrm{Co} / \mathrm{Au}$ trilayer system is not possible, but an accurate representation can be obtained by considering a very thin ferromagnetic film deposited onto a thick noble-metal layer. In this case, the dielectric tensor of the ferromagnetic film is given by

$$
\left(\begin{array}{ccc}
\epsilon & 0 & Q m_{y} \\
0 & \epsilon & 0 \\
-Q m_{y} & 0 & \epsilon
\end{array}\right),
$$

where $\epsilon$ is the dielectric constant of the metallic film and $Q$ is the MO constant. Normally $Q$ is much smaller than $\epsilon$ and, and therefore, the SPP wave vector for the entire air/ ferromagnetic-thin-film/metallic-thick-film structure can be written, up to first order in $Q$, as

$$
k_{\|}=k_{\|}^{0}\left[1+\frac{2 i}{\epsilon^{2}-1}\left(\frac{\epsilon}{1+\epsilon}\right)^{1 / 2}\left(q_{0} d Q m_{y}\right)\right],
$$

where $d$ is the thickness of the ferromagnetic overlayer, and where we assume it has the same dielectric constant as the thick metallic film; $k_{\|}^{0}$ is the SPP wave vector of the structure in the absence of MO activity and $q_{0}=2 \pi / \lambda$.

We note that the SPP wave vector depends linearly on the $y$ component of the magnetization. Then, in the Kretschmann configuration, the angle where the in-plane wave vector of the incident light matches the wave vector of the SPP of the layers (i.e., the reflectivity is minimum) will also depend on 

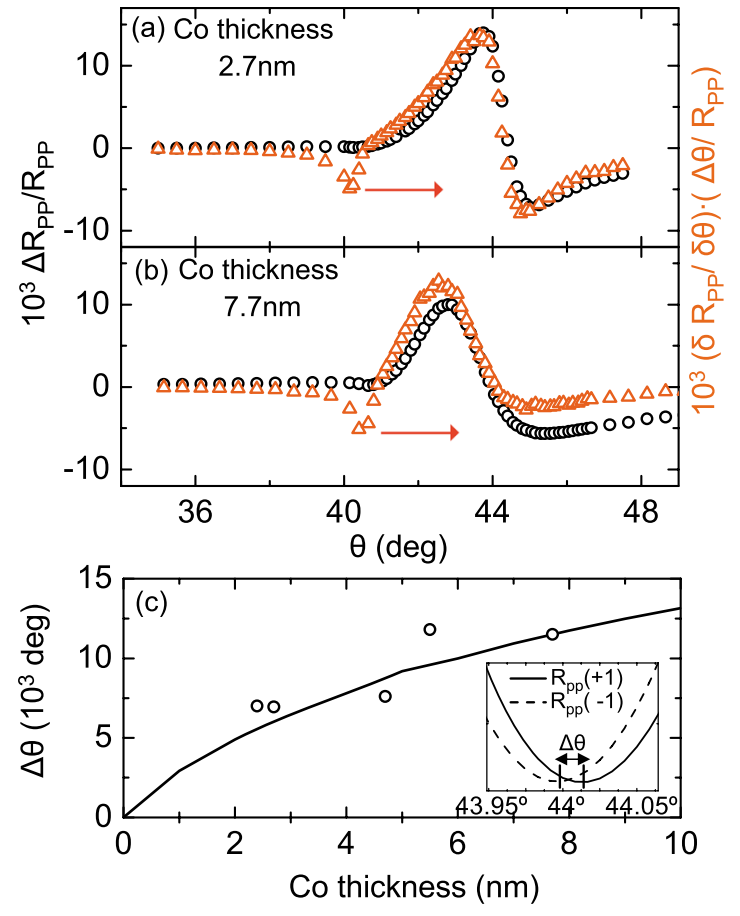

FIG. 2. (Color online) Variation in reflectivity (circles) and the derivative of the reflectivity (triangles) for two different samples with Co thicknesses of (a) 2.7 and (b) $7.7 \mathrm{~nm}$. The sharp dip observed in the derivative of the reflectivity at 40 deg corresponds to the onset of total internal reflection. (c) Angular shift of the minimum in reflectivity as a function of Co thickness. The circles correspond to the experimental values of $\Delta \theta$ as defined in the text. The line is a theoretical calculation. The inset shows the angular shift observed in the $6 \mathrm{~nm}$ thick Co sample.

the $y$ component of the magnetization. Thus, the TMOKE $\left(\Delta R_{p p} / R_{p p}\right)$ is related to the derivative of the reflectivity with respect to the SPP wave vector; in other words to $\left(\delta R_{p p} / \delta \theta\right)\left(\Delta \theta / R_{p p}\right)$, where $\Delta \theta=\theta_{\text {min }}(+1)-\theta_{\min }(-1)$ is the angular shift of the reflectivity minimum when the magnetization is reversed, and represents the variation of the SPP wave vector induced by the change in magnetization direction.

To illustrate this behavior, Fig. 2 shows experimental values for both TMOKE (circles) and $\left(\delta R_{p p} / \delta \theta\right)\left(\Delta \theta / R_{p p}\right)$ (triangles) as function of incident angle for two samples with different Co thicknesses: (a) 2.5 and (b) $7.7 \mathrm{~nm}$. Both curves appear nearly identical; therefore, we conclude that when SPPs are excited the transverse Kerr effect is related to changes in the SPP wave vector. The experimental values of $\Delta \theta$ used in Figs. 2(a) and 2(b) were obtained from the peakto-peak intensity in $\Delta R_{p p} / R_{p p}$. This angular shift is presented in Fig. 2(c) for different samples (circles). Also in the same figure we present the theoretical dependence of $\Delta \theta$ as a function of the Co layer thickness. The optical and magnetooptical constants used in the calculations were obtained from ellipsometry and polar Kerr measurements carried out on thick $\mathrm{Au}$ and $\mathrm{Co}$ layers grown under identical conditions. As can be observed, the variations in the SPP wave vector induced by magnetization changes $(\Delta \theta)$ increase with Co thickness [see Eq. (2)], tuning the magnitude of the nonreciprocity effects in these metallic SPP based structures.
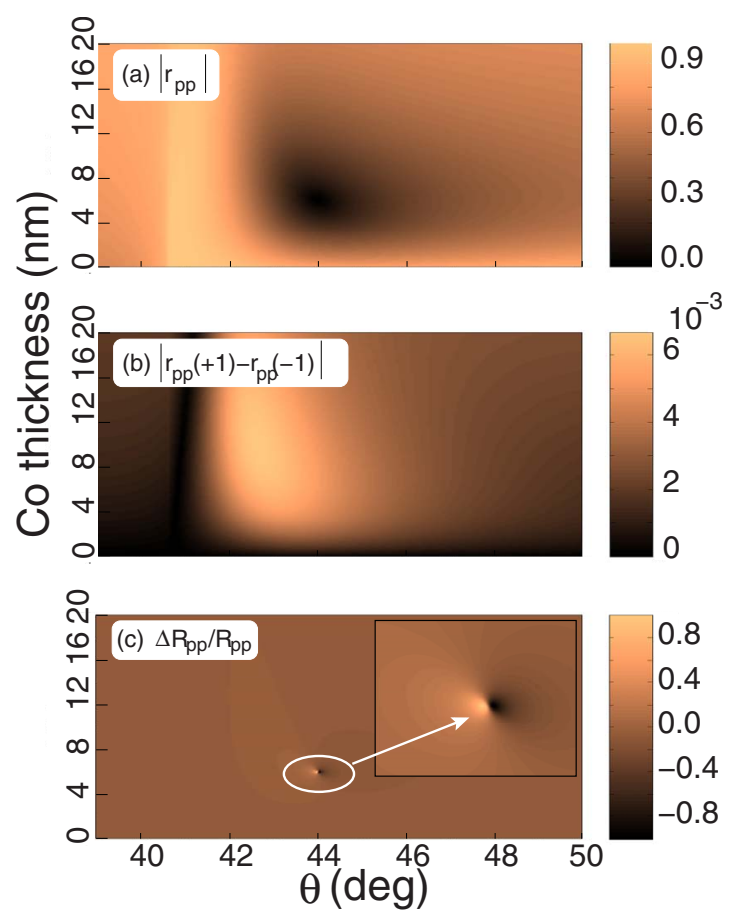

FIG. 3. (Color online) Contour plots of (a) $\left|r_{p p}(0)\right|$, (b) $\left|r_{p p}(+1)-r_{p p}(-1)\right|$, and (c) $\Delta R_{p p} / R_{p p}$ as function of incidence angle and Co thickness in the Kretschmann configuration.

We now focus on the enhancement of the magneto-optical activity when the SPP is excited, and its Co thickness dependence. In Figs. 1(e) and 1(f) we present $\Delta R_{p p} / R_{p p}$ data without and with SPP excitation, respectively, as a function of Co thickness in the range $0-10 \mathrm{~nm}$. In the absence of SPP excitation we find the expected monotonic increase in MO signal as the Co thickness increases. In contrast, when SPPs are excited, the MO activity exhibits a maximum for an approximate Co thickness of $6 \mathrm{~nm}$. This is a rather counterintuitive result since we should expect a similar behavior as that obtained for the magnetic field induced variation of the SPP wave vector [see Fig. 2(c)]: thicker Co layer would be expected to yield a larger MO activity. However, $\Delta R_{p p} / R_{p p}$ is not only related to $\Delta \theta$, which increases with the Co thickness, but also to the derivative of the reflectivity with respect to the incident angle. Also, due to the absorption of the Co layer, as we increase the thickness of the Co layer the reflectivity curve broadens and therefore the magnitude of the derivative decreases. In fact, the Co thickness at which $\Delta R_{p p} / R_{p p}$ reaches a maximum is determined by the Co thickness at which $R_{p p}$ reaches a minimum (which corresponds to the optimum SPP-light coupling conditions). This is better seen in Fig. 3 where we present theoretical contour plots of the optical contribution $\left|r_{p p}(0)\right|$ [Fig. 3(a)], and of the magnetic $\left|r_{p p}(+1)-r_{p p}(-1)\right|$ contribution [Fig. 3(b)] to $\Delta R_{p p} / R_{p p}$ [Fig. 3(c)]:

$$
\frac{\Delta R_{p p}}{R_{p p}}=\frac{\left|r_{p p}(+1)-r_{p p}(-1)\right|}{\left|r_{p p}(0)\right|} \cos \left(\theta_{P}+\theta_{A}\right),
$$

where $r_{p p}\left(m_{y}\right)$ is the complex reflection amplitude, which depends linearly on the $m_{y}$ component of the Co layer mag- 
netization; $\theta_{P}$ and $\theta_{A}$ are phases associated with the nonmagnetic and magnetic parts of the complex reflection amplitude. The sinusoidal term in Eq. (3) determines a sign factor in the range of angles considered.

As can be seen in Fig. 3(a), the optical contribution reaches a maximum at $40.65 \mathrm{deg}$ for any Co thickness (this angle corresponds to the total internal reflection condition), and a minimum appears at $44.11 \mathrm{deg}$ for a Co thickness of $\sim 6 \mathrm{~nm}$. Thus the position of the minimum is controlled by the SPP excitation. The purely magnetic part [Fig. 3(b)] exhibits a much stronger incidence angle dependence than Co thickness dependence. It reaches its maximum for an incidence angle of $42.69 \mathrm{deg}$ at a Co thickness of $9.9 \mathrm{~nm}$. Finally, if we consider the TMOKE signal [Fig. 3(c)] we note that it exhibits an extremely sharp dependence on both Co thickness and incidence angle. It reaches maximum value at $44.1^{\circ}$ and $6 \mathrm{~nm}$ Co thickness. By comparing the three plots, we observe that the leading peak in $\Delta R_{p p} / R_{p p}$ is dominated by the optical part, which depends on optimal SPP excitation, occurring at an angle that depends on the trilayer as a whole and hence on the Co layer thickness.

In summary, we have presented experimental evidence of nonreciprocal magnetic field induced effects in the propagation properties SPP in $\mathrm{Au} / \mathrm{Co} / \mathrm{Au}$ based SPPs. This effect is observed at low magnetic fields and depends on the magnetization of the Co layer and can be tuned by varying the Co layer thickness. In such structures we have also observed a strong increase of magnitude of the TMOKE $\left(\Delta R_{p p} / R_{p p}\right)$ when the SPP is excited. It does not have a monotonic increase as we increase the Co thickness, but reaches a maximum very close to the observed value for optimum SPP excitation and then decreases again. The observed effects could be important in surface plasmon spectroscopy applications, where the ability to modulate the SPP reflectivity signal by means of an external magnetic field can lead to greatly enhanced sensitivity.

Financial support from the Spanish Ministry of Science and Education (NAN2004-09195-C04-01, PR2005-0017, and MAT2005-05524-C02-01), Comunidad de Madrid (Ref. S-0505/MAT/0194) and CSIC (Refs. 200650I130 and 200650I032) are acknowledged. R.C. was supported in part by DOE Grant No. DE-FG02-06ER46273 and by the NSF Frontiers in Physics FOCUS Center.
${ }^{1}$ S. A. Maier and H. A. Atwater, J. Appl. Phys. 98, 011101 (2005).

${ }^{2}$ J.-C. Weeber, Y. Lacroute, and A. Dereux, Phys. Rev. B 68, 115401 (2003).

${ }^{3}$ R. Zia, M. D. Selker, and M. L. Brongersma, Phys. Rev. B 71, 165431 (2005).

${ }^{4}$ T. Nikolajsen, K. Leosson, and S. I. Bozhevolnyi, Appl. Phys. Lett. 85, 5833 (2004).

${ }^{5}$ A. G. Brolo, R. Gordon, B. Leathem, and K. L. Kavanagh, Langmuir 20, 4813 (2004).

${ }^{6}$ I. I. Smolyaninov, J. Elliott, A. V. Zayats, and C. C. Davis, Phys. Rev. Lett. 94, 057401 (2005).

${ }^{7}$ J. J. Brion, R. F. Wallis, A. Hartstein, and E. Burstein, Phys. Rev. Lett. 28, 1455 (1972).

${ }^{8}$ R. E. Wames and W. F. Hall, Phys. Rev. Lett. 29, 172 (1972).

${ }^{9}$ R. F. Wallis, J. J. Brion, E. Burstein, and A. Hartstein, Phys. Rev.
B 9, 3424 (1974).

${ }^{10}$ K. W. Chiu and J. J. Quinn, Phys. Rev. B 5, 4707 (1972).

${ }^{11}$ A. Hartstein and E. Burstein, Solid State Commun. 14, 1223 (1974).

${ }^{12}$ V. I. Safarov, V. A. Kosobukin, C. Hermann, G. Lampel, J. Peretti, and C. Marliere, Phys. Rev. Lett. 73, 3584 (1994).

${ }^{13}$ C. Hermann, V. A. Kosobukin, G. Lampel, J. Peretti, V. I. Safarov, and P. Bertrand, Phys. Rev. B 64, 235422 (2001).

${ }^{14}$ D. P. Kumah, A. Cebollada, C. Clavero, J. M. Garcia-Martin, J. R. Skuza, R. A. Lukaszew, and R. Clarke, J. Phys. D 40, 2699 (2007).

${ }^{15}$ E. Kretschmann, Z. Phys. 241, 313 (1971).

${ }^{16}$ H. Raether, Surface Plasmons on Smooth and Rough Surfaces and on Gratings (Springer-Verlag, Berlin, 1988). 